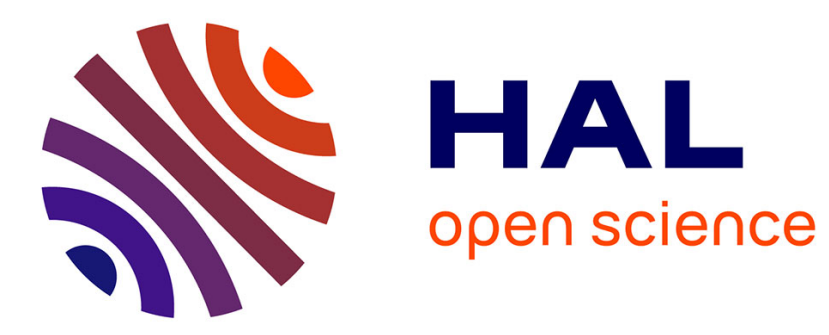

\title{
Asymptotic dynamics of quantum discord in open quantum systems
}

K Berrada, H Eleuch, Y Hassouni

\section{To cite this version:}

K Berrada, H Eleuch, Y Hassouni. Asymptotic dynamics of quantum discord in open quantum systems. Journal of Physics B: Atomic, Molecular and Optical Physics, 2011, 43 (14), pp.145503. 10.1088/0953-4075/44/14/145503 . hal-00636645

\section{HAL Id: hal-00636645 \\ https://hal.science/hal-00636645}

Submitted on 28 Oct 2011

HAL is a multi-disciplinary open access archive for the deposit and dissemination of scientific research documents, whether they are published or not. The documents may come from teaching and research institutions in France or abroad, or from public or private research centers.
L'archive ouverte pluridisciplinaire HAL, est destinée au dépôt et à la diffusion de documents scientifiques de niveau recherche, publiés ou non, émanant des établissements d'enseignement et de recherche français ou étrangers, des laboratoires publics ou privés. 


\title{
Asymptotic Dynamics of Quantum Discord in Open Quantum Systems
}

\author{
K. Berrada ${ }^{\mathbf{1}, \mathbf{2}}$, H. Eleuch ${ }^{\mathbf{3}}$ and Y. Hassouni ${ }^{2}$ \\ (1) Institut für Angewandte Physik, Technische Universität Darmstadt, D-64289, Germany \\ (2) The Abdus Salam International Centre for Theoretical Physics, Trieste, Italy \\ ${ }^{(3)}$ Institute for Quantum Science and Engineering and Department of Physics and Astronomy, \\ Texas A\&M University, College Station, Texas 77843, USA
}

\begin{abstract}
It is well known that quantum entanglement makes possible certain tasks in quantum information theory. However, there are also quantum tasks that display the quantum advantage without entanglement. Distinguishing classical and quantum correlations in quantum systems is therefore of both practical and fundamental importance. Realistic quantum systems are not closed and therefore it is important to study the various correlations when the system loses its coherence due to interactions with the environment. In this paper, we study in detail the dynamics of different kinds of correlations, classical correlation, quantum discord, and entanglement in open quantum systems, in particular a two-qubit system evolving under quantum dynamical semigroups of Kossakowski-type of completely positive maps. In such an environment classical and quantum correlations can even persist asymptotically. By analytic and numerical analysis, we find that the quantum discord is larger than the classical correlation for asymptotic states. Furthermore, we show that the quantum discord is more resistant to the action of the environment than the quantum entanglement and it can persist even in the asymptotic long-time regime.
\end{abstract}

PACS numbers: 03.67.-a, 03.67.Mn, 03.65.Ud

\section{INTRODUCTION}

In the field of quantum information it is important to distinguish between quantum and classical aspects of correlation in a composite quantum state. It is well known that many operations in various quantum information processing tasks depend largely on special kind of quantum correlation, that is, entanglement. Much work has been performed in order to subdivide quantum states into separable and entangled states [1-3]. However, there are other nonclassical correlations apart from entanglement [4-7] that can be of great importance to this field and some of these have been verified experimentally $[8,9]$. These correlations are more general and more fundamental than entanglement. Therefore, it is important to study, characterize and quantify quantum and classical correlations. Several measures of these quantum correlations have been investigated in the literature [10-12], and among them the quantum discord [10] has recently received a great deal of attention [13-19]. Quantum discord has been proposed as the key resource present in certain quantum communication tasks and quantum computational models without containing much entanglement $[7,20,21]$. The quantum discord quantifies nonclassical correlations of more general and more fundamental type than entanglement because separable mixed states (without entanglement) can have nonzero quantum discord. This indicates that classical communication can give rise to quantum correlations due to the existence of nonorthogonal quantum states. For pure entangled states quantum discord coincides with the entanglement entropy. However, for two-qubit mixed states, the relation between the classical and quantum correlations is complicated and not yet clear.

For understanding and distinguishing between classical and quantum correlations, it is important to assume that the total correlation is a directly sum of the both correlations. For a bipartite system $\rho^{a b}$, it is largely accepted that quantum information mutual measures its total correlation (classical and quantum correlation) defined as [22-25]

$$
\mathcal{I}\left(\rho^{a b}\right):=S\left(\rho^{a}\right)+S\left(\rho^{b}\right)-S\left(\rho^{a b}\right),
$$

where $\rho^{a}$ and $\rho^{b}$ are the reduced density matrices of the bipartite state $\rho^{a b}$ and $S(\rho)=-\operatorname{tr}\left(\rho \log _{2} \rho\right)$ is the von Neumann entropy. However, the mutual quantum information may be written as a sum of classical correlation $\mathcal{C}\left(\rho^{a b}\right)$ and quantum correlation, that is, quantum dis$\operatorname{cord} \mathcal{C}\left(\rho^{a b}\right)$ as

$$
\mathcal{I}\left(\rho^{a b}\right):=\mathcal{C}\left(\rho^{a b}\right)+\mathcal{Q}\left(\rho^{a b}\right) .
$$

In order to quantify quantum discord contained in the bipartite state, Ollivier and Zurek [10] have proposed the use of von Neumann type measurements, which consists of a set of one-dimensional projectors that sum up to the identity. The projective measurements on subsystem remove all nonclassical correlations between the parts, i.e., after a measurement on particular subsystem, all quantum correlations are destroyed. If projector measurements $\left\{\Lambda_{j}\right\}$ are performed locally only on the subsystem $b$, then the conditional density operator $\rho_{j}$ with the measurement result $j$ is

$$
\rho_{j}^{a b}=\frac{1}{p_{j}}\left(I \otimes \Lambda_{j}\right) \rho^{a b}\left(I \otimes \Lambda_{j}\right)
$$

where $p_{j}=\operatorname{tr}\left(I \otimes \Lambda_{j}\right) \rho^{a b}\left(I \otimes \Lambda_{j}\right)$ is the probability for the measurement of the $j$ th state and $I$ is the identity operator of subsystem $a$. However, the density matrix 
$\rho_{j}^{a b}$ is considered as a conditional density operator and we can define the quantum conditional entropy with the respect of this measurement [25]

$$
S\left(\rho^{a b} \mid\left\{\Lambda_{j}\right\}\right):=\sum_{j} p_{j} S\left(\rho_{j}\right)
$$

and the related quantum mutual information of this measurement is defined as

$$
\mathcal{I}\left(\rho^{a b} \mid\left\{\Lambda_{j}\right\}\right):=S\left(\rho^{a}\right)-S\left(\rho^{a b} \mid\left\{\Lambda_{j}\right\}\right)
$$

To derive classical correlation, since $\mathcal{I}\left(\rho^{a b} \mid\left\{\Lambda_{j}\right\}\right)$ depends on the projector operators $\left\{\Lambda_{j}\right\}$, we take the maximum of $\mathcal{I}\left(\rho^{a b} \mid\left\{\Lambda_{j}\right\}\right)$, taking into the all possible projectors. On other word, the classical part is defined as the maximal information about subsystem $a$ that can be obtained by performing a measurement on the subsystem $b$. The classical correlation between the two subsystems $a$ and $b$ is defined as

$$
\begin{aligned}
\mathcal{C}\left(\rho^{a b}\right) & :=\sup _{\left\{\Lambda_{j}\right\}} \mathcal{I}\left(\rho^{a b} \mid\left\{\Lambda_{j}\right\}\right) . \\
& =S\left(\rho^{a}\right)-\min _{\left\{\Lambda_{j}\right\}}\left[S\left(\rho^{a b} \mid\left\{\Lambda_{j}\right\}\right)\right] .
\end{aligned}
$$

In this scenario, the quantum discord that measures the nonclassical correlations between two subsystem, can be introduced as a difference between the original quantum mutual information in Eq. (1) and the the classical correlation in Eq. (6) as

$$
\mathcal{Q}\left(\rho^{a b}\right):=\mathcal{I}\left(\rho^{a b}\right)-\mathcal{C}\left(\rho^{a b}\right),
$$

which is always non-negative quantity by expressing mutual information in terms of quantum relative entropy and invoking the monotonicity property of the latter $[26,27]$. The zero-discord states are relatively well study: $\mathcal{Q}\left(\rho^{a b}\right)=0$ if and only if there exists a complete orthonormal basis $\{|k\rangle\}$ for the subsystem $a$ and some density operator $\rho^{b}$ for the subsystem b such that $\rho^{a b}=\sum_{k} p_{k}|k\rangle\langle k| \otimes \rho^{b}$. Recently various methods to detect zero-discord [28-30] have been proposed for a given state as well as for an unknown state [31]. Moreover, it is found that vanishing quantum discord is related to the complete positivity of a map [32] and the local broadcasting of quantum correlations [15].

Besides the characterization and quantification of classical and quantum correlations, another important problem is the behavior of these correlations under the action of decoherence. Every natural object is in contact with its environment, so its dynamics is that of an open system. Thus, the interaction between a composite quantum system and its environment and understanding the dynamics of different kinds of correlations has attracted more interest. The problem of the formulation and characterization of the dynamics correlations of open systems in the quantum regime has a long and extensive history. The quantum entanglement dynamics in open quantum systems was broadly investigated in the literature. However few works dealt with the effect of the environment on quantum discord [33-37].

On the other hand, the definition of quantum discord in Eq. (7) exhibits an optimization problem. However, it is usually intractable to determine the quantum discord for generic cases. Even in the simplest bipartite quantum states, an analytical expression for general two-qubit state is still missing. The dynamics of the classical and quantum correlations in the presence of an environment has been studied for a certain class of high symmetrical states $[33,34,38]$. For this reason the relation between the dynamics of the different kinds of correlations is not known for more general quantum system.

More recently M. Ali et al. [39] have developed an analytic method to evaluate both classical correlations and quantum discord for the complete set of two-qubit $X$ states depending on seven real-valued parameters in order to deepen the understanding the relation between different kinds of correlations. Using this result, we present a detailed analysis of the time evolution of classical and quantum correlations for a large class of two-qubit states in the case of a Lindblad-type master equation. This dynamics that exhibits a rich manifold of asymptotic states that may be more or less classical and quantum correlated with respect to the initial states they emerge from, including maximally entangled Bell states, separable, and nonseparable states. Analytical expressions are calculated and numerically represented, which exhibit the dependence of the classical correlation and quantum discord for asymptotic states. However, it is found that quantum discord can be larger than classical correlation and it can persist even in the asymptotic long-time regime. Furthermore, the comparison between the dynamics of quantum discord and quantum entanglement shown that quantum discord behave differently under the effect of environment. Our observations may have important implications in exploiting these correlations in quantum information processing and transmission.

The paper is organized as follows. In Sec. II, we study the open dynamics of classical and quantum correlations of two qubits in the present model. Analytic derivation of the expression of different kinds of correlations are presented. In Sec. III, we apply this result for a large family of two-qubit states, studying the relation between the dynamics of different kinds of correlations. Finally, we conclude this work in Sec. IV.

\section{DYNAMICS OF THE CLASSICAL AND QUANTUM CORRELATION IN OPEN BIPARTITE SYSTEMS}

In this section, we describe the asymptotic dynamics of two-qubit systems and we derive the classical correlation and quantum discord for them.

Let us consider a bipartite system composed of two qubits immersed in external environment via standard 
weak-coupling limit techniques [40]. The reduced irreversible dynamics is described by one-parameter semigroup of linear maps, which called quantum dynamical semigroups obtained from $\gamma(t)=\exp (t \mathbf{L}))$, and it is gov- erned by the master equation: $\partial_{t} \rho(t)=\mathbf{L}[\rho(t)]$ where the generator $\mathbf{L}$ takes care the effects of the environment through the elements of the Kossakowski matrix [40-42]. Formally, we have

$$
\partial_{t} \rho(t)=\mathbf{L}[\rho(t)]=-i \frac{\Omega}{2}\left[\Sigma_{3}, \rho(t)\right]+\sum_{i, j=1}^{3} A_{i j}\left(\Sigma_{i} \rho(t) \Sigma_{j}-\frac{1}{2}\left\{\Sigma_{j} \Sigma_{i}, \rho(t)\right\}\right)
$$

where $\Omega$ define the system frequency, $\Sigma_{i}:=\sigma_{i} \otimes \mathbf{I}+\mathbf{I} \otimes \sigma_{i}$ is the $2 \times 2$ identity matrix, $\sigma_{i}$ are the Pauli matrices and the matrix

$$
A=\left[A_{i j}\right]=\left(\begin{array}{ccc}
1 & i \alpha & 0 \\
-i \alpha & 1 & 0 \\
0 & 0 & 1
\end{array}\right) \quad \alpha \in \mathbb{R}, \quad \alpha^{2} \leq 1
$$

is positive semi-definite (elements of the Kossakowski matrix) [42]. This shows that the semigroup generated by the master equation consists of completely positive maps $\gamma(t)$ for all $t \geq 0$.

By means of the single qubit Pauli matrices $\sigma_{i}^{(1)}=$ $\sigma_{i} \otimes \mathbf{I}$ and $\sigma_{i}^{(2)}=\mathbf{I} \otimes \sigma_{i}$, we can write the purely dissipative contribution to the generator

$\mathbf{D}[\rho(t)]=\sum_{i, j=1}^{3} A_{i j} \sum_{a, b=1}^{2}\left(\sigma_{i}^{(a)} \rho(t) \sigma_{j}^{(b)}-\frac{1}{2}\left\{\sigma_{j}^{(b)} \sigma_{i}^{(a)}, \rho(t)\right\}\right)$.

In this way there are six Kraus operators $\sigma_{i}^{(a)}, a=1,2$; $i=1,2,3$ and the $6 \times 6$ Kossakowski matrix reads

$$
K=\left[K_{i j}^{(a b)}\right]=\left(\begin{array}{ll}
K^{(11)} & K^{(12)} \\
K^{(21)} & K^{(22)}
\end{array}\right)=\left(\begin{array}{cc}
A & A \\
A & A
\end{array}\right) .
$$

From the theory of open quantum systems [40, 41, 44], the coefficients $K_{i j}^{(a b)}$ in the Kossakowski matrix relative to the $i$-th Pauli matrix of the $a$-th qubit, respectively the $j$-th Pauli matrix of the $b$-th qubit, $a, b=1,2 ; i, j=1,2,3$, are determined by the Fourier transforms of the two-point time-correlation functions with respect to an environment equilibrium state $\omega$, $\omega\left(B_{i}^{(a)} B_{j}^{(b)}(t)\right)$, of the environment operators $B_{i}^{(a)}$ appearing in the system-environment interaction $H_{I}=$ $\sum_{i=1}^{3}\left(\sigma_{i}^{(1)} \otimes B_{i}^{(1)}+\sigma_{i}^{(2)} \otimes B_{i}^{(2)}\right)$. The symmetric form of (3) thus results when both qubits are linearly coupled to bath operators such that: $B_{1,2,3}^{(1)}=B_{1,2,3}^{(2)}=B_{1,2,3}$ and $\omega\left(B_{1,2} B_{3}(t)\right)=0$.

In the following, we are interested to the time evolution of two-qubit system prepared initially in the form

$\rho=a|1\rangle\langle 1|+d| 2\rangle\langle 2|+b| 3\rangle\langle 3|+c| 4\rangle\langle 4|+e| 1\rangle\langle 2|+f| 2\rangle\langle 1|$. where $a, b, c$ and $d$ are real constants satisfying the positive conditions and unit trace, that is, $a+b+c+d=1$, and the set $\{|1\rangle,|2\rangle,|3\rangle,|4\rangle\}$ form an orthonormal basis related to two-qubit standard basis $\{|00\rangle,|01\rangle,|10\rangle,|11\rangle\}$ by

$$
|1\rangle=|00\rangle,|2\rangle=|11\rangle,|3\rangle=\frac{|01\rangle+|10\rangle}{\sqrt{2}},|4\rangle=\frac{|01\rangle-|10\rangle}{\sqrt{2}} .
$$

The density matrix (12) is represented in standard basis as

$$
\rho=\left(\begin{array}{cccc}
a & 0 & 0 & e \\
0 & \frac{b+c}{2} & \frac{b-c}{2} & 0 \\
0 & \frac{b-c}{2} & \frac{b+c}{2} & 0 \\
f & 0 & 0 & d
\end{array}\right)
$$

Equation (14) describes a so-called quantum $X$ state that is entangled if and only if either $\frac{(a+b)^{2}}{4}<e^{2}$ or $a d<\frac{(b-c)^{2}}{4}$. Both conditions cannot hold simultaneously. The density operator (14) includes a very large family of two-qubit states, including Bell states, Werner states, Horodecki states, etc.

The evolution of the density operator $\rho(t)$ initially prepared in Eq. (12) can be obtained as

$$
\begin{aligned}
\rho(t)= & a(t)|1\rangle\langle 1|+d(t)| 2\rangle\langle 2|+b(t)| 3\rangle\langle 3|+c(t)| 4\rangle\langle 4| \\
& +e(t)|1\rangle\langle 2|+f(t)| 2\rangle\langle 1| .
\end{aligned}
$$


where

$$
\begin{aligned}
a(t) & =\frac{(1-\alpha)^{2}}{3+\alpha^{2}} R+\frac{2(1+\alpha) a-(1-\alpha)^{2}(b+d)}{3+\alpha^{2}} E_{+}(t) \\
& +\sqrt{1-\alpha^{2}} \frac{(1+\alpha)^{2} a-2(1-\alpha) d+(1+\alpha)^{2} b}{(1+\alpha)\left(3+\alpha^{2}\right)} E_{-}(t) \\
d(t) & =\frac{(1+\alpha)^{2}}{3+\alpha^{2}} R-\frac{(1+\alpha)^{2} a-2(1+\alpha) d+(1+\alpha)^{2} b}{3+\alpha^{2}} E_{+}(t) \\
& -\sqrt{1-\alpha^{2}} \frac{2(1+\alpha) a-(1-\alpha)^{2}(b+d)}{(1-\alpha)\left(3+\alpha^{2}\right)} E_{-}(t) \\
b(t) & =\frac{(1-\alpha)^{2}}{3+\alpha^{2}} R+\frac{2\left(1+\alpha^{2}\right) b-\left(1-\alpha^{2}\right)(a+d)}{3+\alpha^{2}} E_{+}(t) \\
& +\sqrt{1-\alpha^{2}} \frac{(1+\alpha)^{3} a+(1-\alpha)^{3} d-2\left(1-\alpha^{2}\right) b}{\left(1-\alpha^{2}\right)\left(3+\alpha^{2}\right)} E_{-}(t) \\
c(t) & =c \\
e(t) & =e \exp (-12 t) \\
f(t) & =f \exp (-12 t),
\end{aligned}
$$

with

$$
\begin{aligned}
& R=a+b+d, \quad E_{+}(t)=e^{-8 t} \cosh 4 t \sqrt{1-\alpha^{2}}, \\
& E_{-}(t)=e^{-8 t} \sinh 4 t \sqrt{1-\alpha^{2}} .
\end{aligned}
$$

It is known that in certain specific situations, coupling an environment need not have destroy correlations between subsystems, but can create them, even create correlations that persist at arbitrary long times. This possibility depends on the form of the generator of the reduced dynamics and initial states. Furthermore, in Ref. [43] a necessary and sufficient condition has been obtained for environmental-induced entanglement in an initially separable states for two-qubit system. Starting from an initially separable states, the correlations generated at short times can persist asymptotically, and also starting from initially entangled states, its entanglement content asymptotically increase. This can happen in the present case and the correlation generation capability of the environment is due to the Kossakowski-generator of the reduced dynamics (8).

Now, our primary aim is to evaluate the quantum discord dynamics $\mathcal{Q}(\rho(t))$ defined by Eq. (7) corresponding to above-mentioned cases. This requires us to derive the quantum mutual information dynamics $\mathcal{I}(\rho(t))$ defined by Eq. (1) and classical correlation $\mathcal{C}(\rho(t))$ defined by Eq. (6).

Let us start by evaluating the total correlations, that is quantum mutual information $\mathcal{I}(\rho(t))$, contained in the bipartite state (15). From Eq. (15), the eigenvalues of the density matrix $\rho(t)$ are given by

$$
\begin{aligned}
& \lambda_{1}(t)=\frac{1}{2}\left[(a(t)+d(t))+\sqrt{(a(t)-d(t))^{2}+4 e(t) f(t)}\right] \\
& \lambda_{2}(t)=\frac{1}{2}\left[(a(t)+d(t))-\sqrt{(a(t)-d(t))^{2}+4 e(t) f(t)}\right] \\
& \lambda_{3}(t)=b(t) \\
& \lambda_{4}(t)=c(t) .
\end{aligned}
$$

The mutual quantum information is given by

$$
\mathcal{I}(\rho(t))=S\left(\rho^{a}(t)\right)+S\left(\rho^{b}(t)\right)+\sum_{i=1}^{4} \lambda_{i}(t) \log _{2} \lambda_{i}(t)
$$

where the quantities $S\left(\rho^{a}(t)\right)$ and $S\left(\rho^{b}(t)\right)$ are the marginal entropies of density operator $\rho(t)$. They have the following form

$$
\begin{aligned}
& S\left(\rho^{a}(t)\right)=-\left(\frac{1+a(t)-d(t)}{2}\right) \log _{2}(1+a(t)-d(t)) \\
& -\left(\frac{1+d(t)-a(t)}{2}\right) \log _{2}(1+d(t)-a(t)) \\
& =S\left(\rho^{b}(t)\right) .
\end{aligned}
$$

After evaluating the total correlations $\mathcal{I}(\rho(t))$, we shall compute the evolution classical correlation $\mathcal{C}(\rho(t))$ using von Neumann type measurements. It is known that any von Neumann measurement for subsystem $b$ can be written as [25]

$$
\Lambda_{j}=V \Pi_{j} V^{\dagger} \quad j=0,1,
$$

where $\Pi_{j}=|j\rangle\langle j|$ is the local measurement for qubit $b$ along the computational base $\{|j\rangle\}$ and $V \in S U(2)$ is an unitary operator with unit determinant. After the measurement $\left\{\Lambda_{j}\right\}$, the bipartite state $\rho(t)$ will change to the ensemble $\left\{p_{j}(t), \rho_{j}(t)\right\}$, that is $2 \times 2$ density matrices, where

$$
\rho_{j}(t):=\frac{1}{p_{j}(t)}\left(I \otimes \Lambda_{j}\right) \rho(t)\left(I \otimes \Lambda_{j}\right)
$$

and $p_{j}(t)=\operatorname{tr}\left(\left(I \otimes \Lambda_{j}\right) \rho(t)\left(I \otimes \Lambda_{j}\right)\right)$. The operator $V$ can be written, up to a constant phase, as

$$
V=t I+i \vec{y} \cdot \vec{\sigma}
$$

With $t, y_{1}, y_{2}, y_{3} \in \mathbb{R}$ and $t^{2}+y_{1}^{2}+y_{2}^{2}+y_{3}^{2}=1$. This implies that these parameters, three among them independent, assuming their values in the interval $[-1,1]$, that is, $t, y_{i} \in[-1,1]$.

In order to obtain the evolution of classical correlation, We need to evaluate $\rho_{j}(t)$ and $p_{j}(t)$. In this way, Eq. (A1) can be rewritten as

$$
p_{j}(t) \rho_{j}(t)=\left(I \otimes\left(V \Pi_{j} V^{\dagger}\right)\right) \rho(t)\left(I \otimes\left(V \Pi_{j} V^{\dagger}\right)\right)
$$


and the minimization over von Neumann measurements $\min _{\left\{\Lambda_{j}\right\}}\left[S\left(\rho(t) \mid\left\{\Lambda_{j}\right\}\right)\right]$ is straight forwardly derived by the analytically method in Ref. [39] and is given as

$$
\min _{\left\{\Lambda_{j}\right\}}\left[S\left(\rho(t) \mid\left\{\Lambda_{j}\right\}\right)\right]=\min \left[f_{\frac{1}{2}, m}(t), f_{1, m}(t)\right] .
$$

This result, which we prove in Appendix $A$, helps to explicitly solve the minimization problem. Then, the evolution of the classical correlation can be expressed as

$$
\mathcal{C}(\rho(t))=S\left(\rho^{a}(t)\right)-\min \left[f_{\frac{1}{2}, m}(t), f_{1, m}(t)\right] .
$$

Therefore, the quantum discord dynamics can be written as

$$
\mathcal{Q}(\rho(t))=\mathcal{I}(\rho(t))-S\left(\rho^{a}(t)\right)+\min \left[f_{\frac{1}{2}, m}(t), f_{1, m}(t)\right]
$$

where the amount of the total correlation $\mathcal{I}(\rho(t))$ is given in Eq. (19).

The above expression exhibits the time evolution of the quantum discord for open bipartite quantum system characterized by a quantum dynamical one-parameter semigroup of completely positive linear maps.
In order to compare the quantum discord dynamics with the entanglement dynamics in the present model, we use the concurrence as a measure of entanglement. For two-qubit states, the concurrence dynamics can be given as [45]

$$
C(\rho(t))=\max \left\{\theta_{1}(t)-\theta_{2}(t)-\theta_{3}(t)-\theta_{4}(t), 0\right\},
$$

where $\theta_{i}(i=1,2,3,4)$ are the square roots of the eigenvalues of the non-Hermitian matrix $\rho(t) \tilde{\rho}(t)$ in descending order. Note that each $\theta_{i}(t)$ is a positive real number and $\tilde{\rho}(t)$ being the spin-flip operation of $\rho(t)$ given by

$$
\tilde{\rho}(t)=\left(\sigma_{y} \otimes \sigma_{y}\right) \rho^{*}(t)\left(\sigma_{y} \otimes \sigma_{y}\right)
$$

where $\rho^{*}(t)$ denotes the complex conjugate of $\rho(t)$. For a two-qubit with a structure defined as in Eq. (15) we have

$$
\tilde{\rho}(t)=\left(\begin{array}{cccc}
a(t) & 0 & 0 & e(t) \\
0 & \frac{(b(t)+c(t)}{2)} & \frac{(b(t)-c(t)}{2)} & 0 \\
0 & \frac{(b(t)-c(t)}{2)} & \frac{(b(t)+c(t)}{2)} & 0 \\
f(t) & 0 & 0 & d(t)
\end{array}\right)
$$

$$
\rho(t) \tilde{\rho}(t)=\left(\begin{array}{cccc}
a(t) d(t)+e^{2}(t) & 0 & 0 & a(t) f(t)+e(t) a(t) \\
0 & \frac{b^{2}(t)+c^{2}(t)}{2} & \frac{b^{2}(t)-c^{2}(t)}{2} & 0 \\
0 & \frac{b^{2}(t)-c^{2}(t)}{2} & \frac{b^{2}(t)+c^{2}(t)}{2} & 0 \\
f(t) d(t)+d(t) e(t) & 0 & 0 & a(t) d(t)+f^{2}(t)
\end{array}\right) .
$$

and therefore the corresponding concurrence can be given as

$$
C(\rho(t))=\max \left\{0, \Xi_{1}(\rho(t)), \Xi_{2}(\rho(t))\right\}
$$

with $\Xi_{1}(\rho(t))=2 e(t)-b(t)-c(t)$ and $\Xi_{2}(\rho(t))=$ $|b(t)-c(t)|-2 \sqrt{a(t) d(t)}$.

In what follows one wants to inquire the relationship between the dynamics of the classical and quantum correlations. In particular, one wants to understand the changes in behaviour in the asymptotic dynamics of different kinds of correlations.

\section{RELATION BETWEEN CLASSICAL CORRELATION, QUANTUM DISCORD AND ENTANGLEMENT IN OPEN QUANTUM SYSTEMS}

In this section, we study in detail the relation between the asymptotic dynamics of the classical correlation, quantum discord, and entanglement for a large family of initial states.
To explore the influence of decoherence on the dynamical behavior of the classical and quantum correlations in the present model, we have plotted the time evolution of $\mathcal{C}(\rho(t)), \mathcal{Q}(\rho(t))$, and $C(\rho(t))$ as a function of time $t$ for various values of parameter $\alpha$ in several classes of twoqubit states. The choice of the range of the values for the plot axes was made only for graphic reasons to make the plot clear. Changing the values of the parameter $\alpha$ does not alter the results of the evolution of the classical and quantum correlations.

Case 1. As a first example, we consider an initial pure separable state, that is, $\rho=|1\rangle\langle 1|$. The state $\rho$ is uncorrelated with zero classical and quantum correlations and it goes into a mixed separable state (32):

$$
\rho(\infty)=\frac{(1-\alpha)^{2}}{3+\alpha^{2}}|1\rangle\left\langle 1\left|+\frac{(1+\alpha)^{2}}{3+\alpha^{2}}\right| 2\right\rangle\left\langle 2\left|+\frac{\left(1-\alpha^{2}\right)}{3+\alpha^{2}}\right| 3\right\rangle\langle 3| .
$$

For this particular case, the different kinds of correlations 
are given as

$$
\begin{aligned}
& \mathcal{C}(\rho(\infty))=S\left(\rho^{a}(\infty)\right)-f_{\frac{1}{2}, 0}(\infty) \\
& \mathcal{Q}(\rho(\infty))=S\left(\rho^{a}(\infty)\right)+f_{\frac{1}{2}, 0}(\infty)-S(\rho(\infty)) \\
& C(\rho(\infty))=0,
\end{aligned}
$$

where the entropies $S\left(\rho^{a}(\infty)\right)$ and $S(\rho(\infty))$ are given by Eqs. (19) and (20) respectively, and the minimal value of the function $f$ reads

$$
\begin{aligned}
f_{\frac{1}{2}, 0}(\infty) & =-\frac{1-\Gamma(\infty)}{2} \log _{2} \frac{1-\Gamma(\infty)}{2} \\
& -\frac{1+\Gamma(\infty)}{2} \log _{2} \frac{1+\Gamma(\infty)}{2}
\end{aligned}
$$

where

$$
\Gamma(\infty)=\left[(a(\infty)-d(\infty))^{2}+b^{2}(\infty)\right]^{\frac{1}{2}} .
$$

Figure 1 displays the asymptotic dynamics of the classical correlation, quantum discord, and concurrence for the pure state $\rho$ for $\alpha=0.5$. The solid line presents the dynamics of quantum discord, the dotted line is for the classical correlation. It can be seen that the dissipative time evolution is able to generate the quantum discord and classical correlation for the state $\rho$, contrariwise the quantum entanglement is zero at any time. Moreover, quantum discord is observed to be larger than classical correlation.

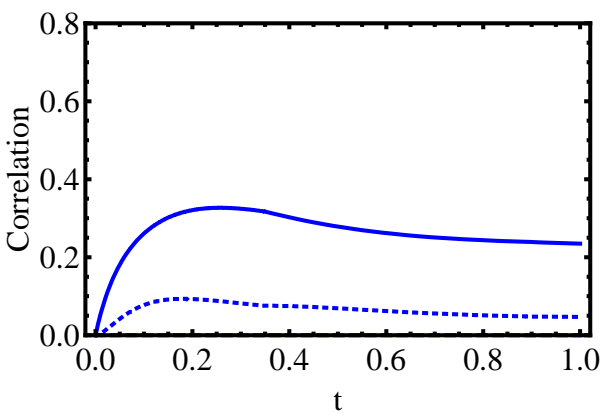

FIG. 1. Asymptotic classical and quantum correlations. Quantum discord (solid line), Classical correlation (dotted line), and $C=0$ with $\alpha=0.5$.

Case 2. We consider an initial maximally entangled pure state, one of the four Bell states defined as $\rho_{1}^{+}=\left|\phi^{+}\right\rangle\left\langle\phi^{+}\right|=1 / 2(|1\rangle\langle 1|+| 2\rangle\langle 2|+| 2\rangle\langle 1|+| 1\rangle\langle 2|)$, $\rho_{1}^{-}=\left|\phi^{-}\right\rangle\left\langle\phi^{-}\right|=1 / 2(|1\rangle\langle 1|+| 2\rangle\langle 2|-| 2\rangle\langle 1|-| 1\rangle\langle 2|)$ $\rho_{2}^{+}=\left|\psi^{+}\right\rangle\left\langle\psi^{+}|=| 3\right\rangle\langle 3|$, and $\rho_{2}^{-}\left|\psi^{-}\right\rangle\left\langle\psi^{-}\right|=$ $|4\rangle\langle 4|$, with $\left|\phi^{ \pm}\right\rangle=1 / \sqrt{2}(|00\rangle \pm|11\rangle)$ and $\left|\psi^{ \pm}\right\rangle=$ $1 / \sqrt{2}(|01\rangle \pm|10\rangle)$. It is known that for any Bell states, we have

$$
\mathcal{C}(\rho(0))=\mathcal{Q}(\rho(0))=C(\rho(0))=1 \text {. }
$$

For this example, classical correlation, quantum discord and any measure of entanglement coincide and are equal to the maximum value of the correlation between subsystems.

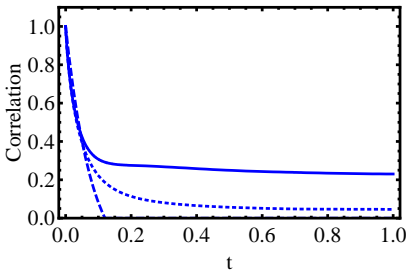

(a)

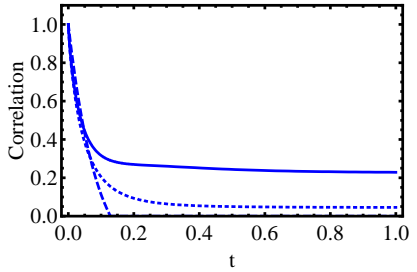

(b)
FIG. 2. Asymptotic classical and quantum correlations for Bell states. Quantum discord (solid line), classical correlation (dotted line), and concurrence (dashed line) with $\alpha=0.5$. (a) for $\rho_{1}^{ \pm}$and $(b)$ for $\rho_{2}^{+}$.

Under the irreversible time evolution, the initial maximal pure states $\rho_{1}^{ \pm}$and $\rho_{2}^{+}$go into an asymptotic mixed separable state which is less classical and quantum correlated :

$\rho(\infty)=\frac{(1-\alpha)^{2}}{3+\alpha^{2}}|1\rangle\left\langle 1\left|+\frac{(1+\alpha)^{2}}{3+\alpha^{2}}\right| 2\right\rangle\left\langle 2\left|+\frac{\left(1-\alpha^{2}\right)}{3+\alpha^{2}}\right| 3\right\rangle\langle 3|$.

In Fig. 2, we plot the dynamics of the correlations for the Bell states $\rho_{1}^{ \pm}$and $\rho_{2}^{+}$. From the figure, the dissipative time evolution shows a sudden death of quantum entanglement, that is, the concurrence vanishes at finite time. On the other hand, as we can see, contrary to what happens to entanglement, the quantum discord may remain constant for long times, that is the case when quantum coherence is alive. This implies that quantum discord is more resistant to noise than entanglement. Moreover, we see that the discord is always greater than classical correlation.

In contrast to previous maximal pure states, the classical and quantum correlations of the state $\rho_{2}^{-}$are unaffected under decoherence and we have

$$
\rho_{2}^{-}(\infty)=|4\rangle\langle 4|,
$$

with the corresponding correlations

$$
\mathcal{C}\left(\rho_{2}^{-}(t)\right)=\mathcal{Q}\left(\rho_{2}^{-}(t)\right)=C\left(\rho_{2}^{-}(t)\right)=1, \quad \forall t \geq 0
$$

This is due to rotational invariance of $|4\rangle$.

Case 3. We take an initial mixed separable state, that is, $\rho=1 / 2(|3\rangle\langle 3|+| 4\rangle\langle 4|)$, with vanishing values of quantum correlations $(\mathcal{Q}(\rho(0))=C(\rho(0)=0)$ and maximal classical correlation $(\mathcal{C}(\rho(0))=1$, all correlations are classical). During the time evolution, the state $\rho$ goes into an asymptotic state with more quantum correlations and less classical correlation :

$$
\begin{aligned}
\rho(\infty)= & \frac{1}{2} \frac{(1-\alpha)^{2}}{\left(3+\alpha^{2}\right)}|1\rangle\left\langle 1\left|+\frac{1}{2} \frac{(1+\alpha)^{2}}{\left(3+\alpha^{2}\right)}\right| 2\right\rangle\langle 2| \\
& +\frac{1}{2} \frac{\left(1-\alpha^{2}\right)}{\left(3+\alpha^{2}\right)}|3\rangle\left\langle 3\left|+\frac{1}{2}\right| 4\right\rangle\langle 4|,
\end{aligned}
$$


with the corresponding correlations

$$
\begin{aligned}
& \mathcal{C}(\rho(\infty))=S\left(\rho^{a}(\infty)\right)-f_{\frac{1}{2}, 0}(\infty) \\
& \mathcal{Q}(\rho(\infty))=S\left(\rho^{a}(\infty)\right)+f_{\frac{1}{2}, 0}(\infty)-S(\rho(\infty)) \\
& C(\rho(\infty))=\frac{2 \alpha^{2}}{3+\alpha^{2}}
\end{aligned}
$$

where the minimal value of the function $f$ is obtained as

$$
\begin{aligned}
f_{\frac{1}{2}, 0}(\infty)= & -\frac{1-\gamma(\infty)}{2} \log _{2} \frac{1-\gamma(\infty)}{2} \\
& -\frac{1+\gamma(\infty)}{2} \log _{2} \frac{1+\gamma(\infty)}{2}
\end{aligned}
$$

with

$$
\gamma(\infty)=\left[(a(\infty)-d(\infty))^{2}+(b(\infty)-c)^{2}\right]^{\frac{1}{2}} .
$$

The dynamics of the classical correlation, quantum discord, and concurrence have been plotted in Fig. 3 for $\rho$ against $t$. The solid line is for quantum discord, the dashed line is for concurrence, and dotted line is for classical correlation. Interestingly, in this particular case, we note that the dissipative time evolution is able to generate the quantum correlations. However, we see that the correlations have a different order as functions of $t$, with classical correlation initially larger than discord and concurrence, but after some time, discord becomes larger than concurrence and classical correlation. Moreover, the all correlations remain constant as time become large.

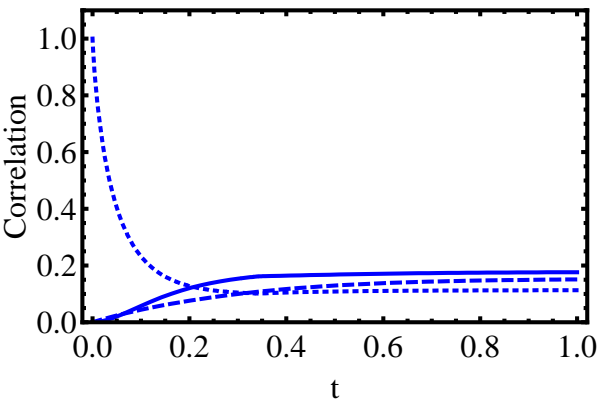

FIG. 3. Asymptotic classical and quantum correlations. Quantum discord (solid line), classical correlation (dotted line), and concurrence (dashed line) with $\alpha=0.5$.

Case 4. Let us consider an initial mixed entangled state, that is, $\rho=1 / 10(3|2\rangle\langle 2|+| 3\rangle\langle 3|+6| 4\rangle\langle 4|)$ with concurrence grater than quantum discord and classical correlation $(C(\rho(0))=0.5, \mathcal{Q}(\rho(0))=$ 0.285829 , and $\mathcal{C}(\rho(0))=0.286845)$. During the process of decoherence, the state $\rho$ goes into an asymptotic mixed entangled state:

$$
\begin{aligned}
\rho(\infty)= & \frac{2}{5} \frac{(1-\alpha)^{2}}{\left(3+\alpha^{2}\right)}|1\rangle\left\langle 1\left|+\frac{2}{5} \frac{(1+\alpha)^{2}}{\left(3+\alpha^{2}\right)}\right| 2\right\rangle\langle 2| \\
& +\frac{2}{5} \frac{\left(1-\alpha^{2}\right)}{\left(3+\alpha^{2}\right)}|3\rangle\left\langle 3\left|+\frac{3}{5}\right| 4\right\rangle\langle 4|,
\end{aligned}
$$

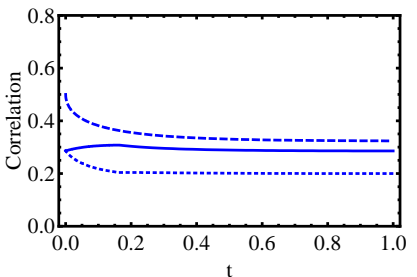

(a) (b)

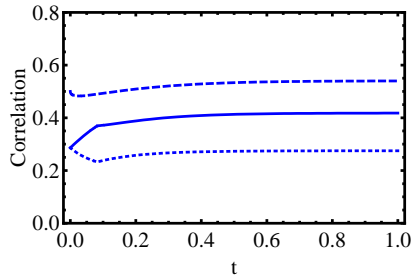

FIG. 4. Asymptotic classical and quantum correlations. Quantum discord (solid line), classical correlation (dotted line), and concurrence (dashed line). ( $a$ ) for $\alpha=0.5$ and $(b)$ for $\alpha=0.9$.

with the corresponding classical and quantum correlations

$$
\begin{aligned}
& \mathcal{C}(\rho(\infty))=S\left(\rho^{a}(\infty)\right)-f_{\frac{1}{2}, 0}(\infty) \\
& \mathcal{Q}(\rho(\infty))=S\left(\rho^{a}(\infty)\right)+f_{\frac{1}{2}, 0}(\infty)-S(\rho(\infty)) \\
& C(\rho(\infty))=\frac{3\left(1+3 \alpha^{2}\right)}{5\left(3+\alpha^{2}\right)}
\end{aligned}
$$

where

$$
\begin{aligned}
f_{\frac{1}{2}, 0}(\infty)= & -\frac{1-\gamma(\infty)}{2} \log _{2} \frac{1-\gamma(\infty)}{2} \\
& -\frac{1+\gamma(\infty)}{2} \log _{2} \frac{1+\gamma(\infty)}{2}
\end{aligned}
$$

with

$$
\gamma(\infty)=\left[(a(\infty)-d(\infty))^{2}+(b(\infty)-c)^{2}\right]^{\frac{1}{2}} .
$$

In Fig. 4 we depict the graphs of the dynamics of the classical and quantum correlations for the state $\rho$. In contrast to previous examples, the dynamic behavior of the different kinds of correlations depends on the parameter $\alpha$. As we can see in Fig. 4, the correlations of the asymptotic state can be larger or smaller than initial one depending on the parameter $\alpha$. If we take for example $\alpha=0.5$, then $\mathcal{C}(\rho(\infty))<0.286845, \mathcal{Q}(\rho(\infty))<0.285829$, and $C(\rho(\infty))<0.5$, i.e. the asymptotic state has less correlation than the initial state (Fig. 4(a)). Whereas for $\alpha=0.9$, the initial entanglement and classical correlation first diminish and then increase again, leading to an asymptotic state with more correlations than initial one. Moreover, it can be seen that for this particular initial state, quantum discord is always less than concurrence but always greater than the classical correlation (Fig. 4(b)).

Case 5. We take an initial two-qubit mixed entangled state, that is, $\rho=1 / 10(5|2\rangle\langle 2|+| 3\rangle\langle 3|+4| 4\rangle\langle 4|)$, with entanglement grater than quantum discord and classical correlation $(C(\rho(0))=0.3, \mathcal{Q}(\rho(0))=$ 0.139036 , and $\mathcal{C}(\rho(0))=0.122556)$. During the time evolution, the state $\rho$ goes into an asymptotic mixed state with less or more quantum correlations depending on the 


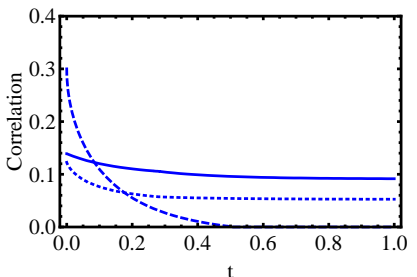

(a)

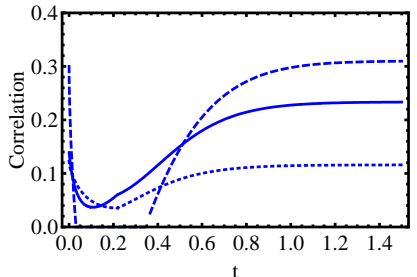

(b)
FIG. 5. Asymptotic classical and quantum correlations. Quantum discord (solid line), classical correlation (dotted line), and concurrence (dashed line). ( $a$ ) for $\alpha=0.5$ and $(b)$ for $\alpha=-0.9$.

parameter $\alpha$ :

$$
\begin{aligned}
\rho(\infty)= & \frac{3}{5} \frac{(1-\alpha)^{2}}{\left(3+\alpha^{2}\right)}|1\rangle\left\langle 1\left|+\frac{3}{5} \frac{(1+\alpha)^{2}}{\left(3+\alpha^{2}\right)}\right| 2\right\rangle\langle 2| \\
& +\frac{3}{5} \frac{\left(1-\alpha^{2}\right)}{\left(3+\alpha^{2}\right)}|3\rangle\left\langle 3\left|+\frac{2}{5}\right| 4\right\rangle\langle 4| .
\end{aligned}
$$

with the corresponding correlations

$$
\begin{gathered}
\mathcal{C}(\rho(\infty))=S\left(\rho^{a}(\infty)\right)-f_{\frac{1}{2}, 0}(\infty) \\
\mathcal{Q}(\rho(\infty))=S\left(\rho^{a}(\infty)\right)+f_{\frac{1}{2}, 0}(\infty)-S(\rho(\infty)) \\
\left\{\begin{array}{ccc}
C(\rho(\infty))=0 & \text { for } \quad \alpha^{2} \leq \frac{3}{11} \\
C(\rho(\infty))=\frac{\left(11 \alpha^{2}-3\right)}{5\left(3+\alpha^{2}\right)} & \text { for } & \frac{3}{11}<\alpha^{2} \leq 1,
\end{array}\right.
\end{gathered}
$$

where

$$
\begin{aligned}
f_{\frac{1}{2}, 0}(\infty)= & -\frac{1-\gamma(\infty)}{2} \log _{2} \frac{1-\gamma(\infty)}{2} \\
& -\frac{1+\gamma(\infty)}{2} \log _{2} \frac{1+\gamma(\infty)}{2}
\end{aligned}
$$

with

$$
\gamma(\infty)=\left[(a(\infty)-d(\infty))^{2}+(b(\infty)-c)^{2}\right]^{\frac{1}{2}} .
$$

In Fig. 5, we display the dynamics of the classical correlation, quantum discord, and concurrence versus $t$ for different values of $\alpha$. Depending on the choice of $\alpha$, we can see that the correlations go into a state with less or more quantum correlations than initial one. On other hand, the dissipative time evolution shows a sudden death of the quantum entanglement, that is, the concurrence vanishes at finite time, and also it exhibits a sudden birth (Fig. 5(b)). Contrary to what happens to entanglement, the discord remains constant for a long time. More interestingly, the quantum discord dominates the classical correlation in this limit.

Case 6. we consider an initial state, that is, Werner state $[46]$

$$
\rho=x\left|\Psi^{-}\right\rangle\left\langle\Psi^{-}\right|+\frac{(1-x)}{4} I
$$

where $\left|\Psi^{-}\right\rangle=1 / \sqrt{2}(|01\rangle-|10\rangle)$ is a maximally entangled state and $0 \leq x \leq 1$.

The parameters defined in the density matrix (14) are given as

$$
\begin{aligned}
& a=b=d=\frac{1-x}{4} \\
& c=\frac{1+3 x}{4} \\
& e=f=0 .
\end{aligned}
$$

The initial Werner state, has a particular property is that the minimization of the function $f$ does not depend on the parameters $k$ and $m$, and we have

$$
\begin{aligned}
& \mathcal{C}(\rho(0))=\frac{1-x}{2} \log _{2}(1-x)+\frac{1-x}{2} \log _{2}(1-x) \\
& \mathcal{Q}(\rho(0))=\frac{1}{4}\left[(1-x) \log _{2}(1-x)+(1+3 x) \log _{2}(1+3 x)\right. \\
& \left.-2(1+x) \log _{2}(1+x)\right] \\
& C(\rho(0))=\max \left\{0, \frac{3 x-1}{2}\right\} .
\end{aligned}
$$

We display these correlations versus $x$ for this state in Fig. 6, we can see that the correlations have a different order depending the parameter $x$ with quantum discord initially larger than concurrence and the classical correlation when $x \lesssim 0.5234$, but for $x>0.5234$ the concurrence becomes larger than quantum discord and the classical correlation.

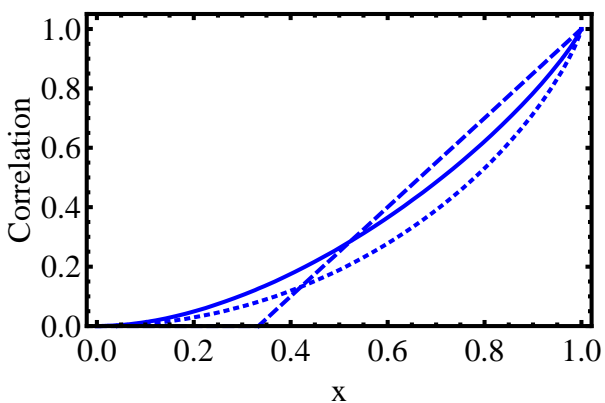

FIG. 6. Classical and quantum correlations for the initial Werner state. Quantum discord (solid line), classical correlation (dotted line), and concurrence (dashed line).

During the process of decoherence, the initial state goes into an asymptotic mixed state with more correlations depending on the parameters $x$ and $\alpha$ :

$$
\begin{aligned}
\rho(\infty)= & \frac{3}{4} \frac{(1+x)(1-\alpha)^{2}}{\left(3+\alpha^{2}\right)}|1\rangle\left\langle 1\left|+\frac{3}{4} \frac{(1+x)(1+\alpha)^{2}}{\left(3+\alpha^{2}\right)}\right| 2\right\rangle\langle 2| \\
& +\frac{3}{4} \frac{(1+x)\left(1-\alpha^{2}\right)}{\left(3+\alpha^{2}\right)}|3\rangle\left\langle 3\left|+\frac{(1+3 x)}{4}\right| 4\right\rangle\langle 4|,
\end{aligned}
$$

with the corresponding classical and quantum correlations

$$
\begin{aligned}
& \mathcal{C}(\rho(\infty))=S\left(\rho^{a}(\infty)\right)-f_{\frac{1}{2}, 0}(\infty) \\
& \mathcal{Q}(\rho(\infty))=S\left(\rho^{a}(\infty)\right)+f_{\frac{1}{2}, 0}(\infty)-S(\rho(\infty))
\end{aligned}
$$




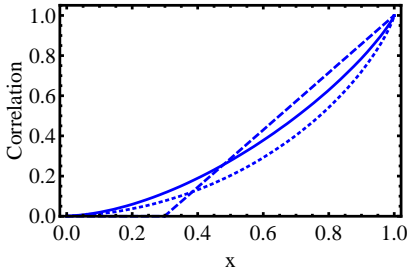

(a)

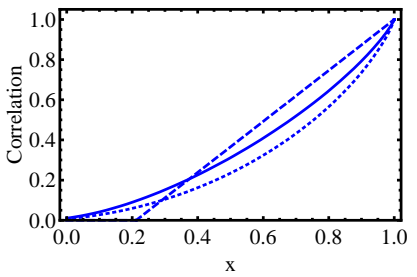

(c)

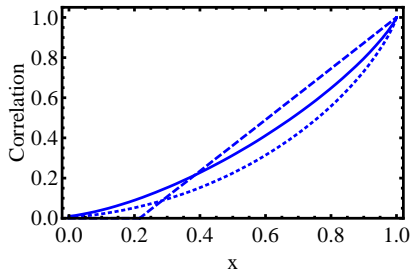

(b)

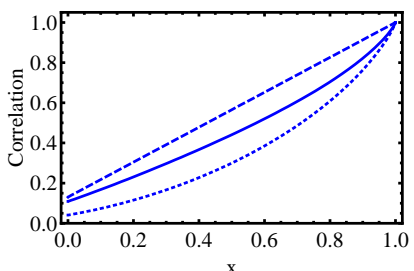

(d)

FIG. 7. Asymptotic classical and quantum correlations for the initial Werner state with (a) $\alpha=0.5 ; t=0.2$, (b) $\alpha=0.5 ; t=1$, (c) $\alpha=0.9$; $t=0.2$, and (d) $\alpha=0.9 ; t=1$. Quantum discord (solid line), classical correlation (dotted line), and concurrence (dashed line).

$$
\left\{\begin{array}{cll}
C(\rho(\infty))=0 & \text { for } & \alpha^{2} \leq \frac{3-9 x}{5-3 x} \\
C(\rho(\infty))=\frac{(5-3 x) \alpha^{2}+9 x-3}{2\left(3+\alpha^{2}\right)} & \text { for } & \frac{3-9 x}{5-3 x}<\alpha^{2} \leq 1,
\end{array}\right.
$$

where the minimal value of the function $f$ is given by

$$
\begin{aligned}
f_{\frac{1}{2}, 0}(\infty)= & -\frac{1-\gamma(\infty)}{2} \log _{2} \frac{1-\gamma(\infty)}{2} \\
& -\frac{1+\gamma(\infty)}{2} \log _{2} \frac{1+\gamma(\infty)}{2}
\end{aligned}
$$

with

$$
\gamma(\infty)=\left[(a(\infty)-d(\infty))^{2}+(b(\infty)-c)^{2}\right]^{\frac{1}{2}} .
$$

In Fig. 7, we display the variation of different types of correlations against $x$ for different values of $t$. From the figure it can be seen that for this particular initial state, the behavior of the correlations is the same as the initial ones. The correlations have different order depending on the values of the parameter $\alpha$ and $x$.

In Fig. 8, we show the time evolution of classical and quantum correlations for the initial Werner states with different values of the parameters $\alpha$ and $x$. We can see that the quantum discord can be smaller or larger than quantum entanglement in the asymptotic limit. Moreover, the quantum discord is always greater than the classical correlation.

Case 7. Finally we consider an initial Horodecki state [47] defined as a mixture of a maximally entangled state, say the Bell state $\left|\Psi^{+}\right\rangle=1 / \sqrt{2}(|00\rangle+|11\rangle)$, and a separable state orthogonal to it, see $|00\rangle$ :

$$
\rho=x\left|\Psi^{+}\right\rangle\left\langle\Psi^{+}|+(1-x)| 00\right\rangle\langle 00|,
$$

where the parameter $x \in[0,1]$. The different elements in

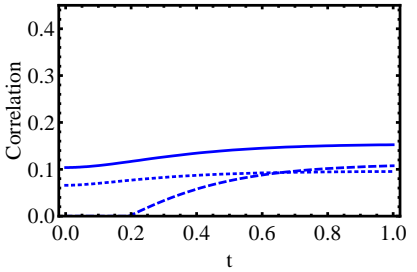

(a)

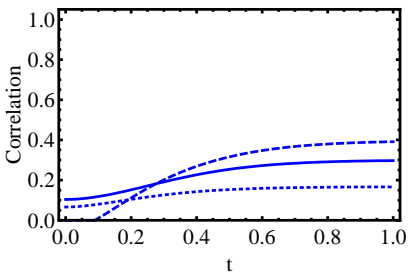

(c)

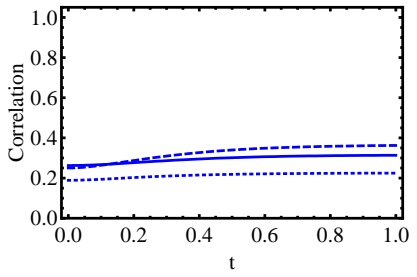

(b)

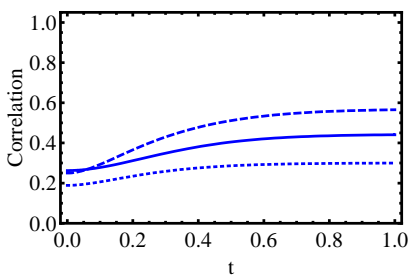

(d)
FIG. 8. Asymptotic classical and quantum correlations for the initial Werner state with (a) $\alpha=0.5 ; x=0.3$, (b) $\alpha=0.5 ; x=0.5$, (c) $\alpha=0.9 ; x=0.3$, and (d) $\alpha=0.9 ; x=0.5$. Quantum discord (solid line), classical correlation (dotted line), and concurrence (dashed line).

the matrix (14) are given as

$$
\begin{aligned}
& a=1-x \\
& b=x \\
& c=d=e=f=0 .
\end{aligned}
$$

In this case, the minimum of the function $f$ is attained for $k=\frac{1}{2}$, that is, $f_{\frac{1}{2}, 0}(0)=f_{\frac{1}{2}, \frac{1}{4}}(0)$. Therefore, The different correlations are given as

$$
\begin{aligned}
& \mathcal{C}(\rho(0))=S\left(\rho^{a}(0)\right)-f_{\frac{1}{2}, 0}(0) \\
& \mathcal{Q}(\rho(0))=S\left(\rho^{a}(0)\right)+f_{\frac{1}{2}, 0}(0)-S(\rho(0)) \\
& C(\rho(0))=x
\end{aligned}
$$

where

$S\left(\rho^{a}(0)\right)=-\frac{x}{2} \log _{2} \frac{x}{2}-\frac{2-x}{2} \log _{2} \frac{2-x}{2}$

$f_{\frac{1}{2}, 0}(0)=-\frac{1-\sqrt{x^{2}+(1-x)^{2}}}{2} \log _{2} \frac{1-\sqrt{x^{2}+(1-x)^{2}}}{2}$

$-\frac{1+\sqrt{x^{2}+(1-x)^{2}}}{2} \log _{2} \frac{1+\sqrt{x^{2}+(1-x)^{2}}}{2}$

$S(\rho(0))=-x \log _{2} x-(1-x) \log (1-x)$.

In Fig. 9, we plot these correlations for various values of $x$. We can see that for the initial Horodecki state, the quantum discord is always greater than classical correlation but always small than the concurrence.

During the time evolution, the initial Horodecki state goes into an asymptotic mixed state with less correlations than initial one:

$\rho(\infty)=\frac{(1-\alpha)^{2}}{3+\alpha^{2}}|1\rangle\left\langle 1\left|+\frac{(1+\alpha)^{2}}{3+\alpha^{2}}\right| 2\right\rangle\left\langle 2\left|+\frac{\left(1-\alpha^{2}\right)}{3+\alpha^{2}}\right| 3\right\rangle\langle 3|$. 


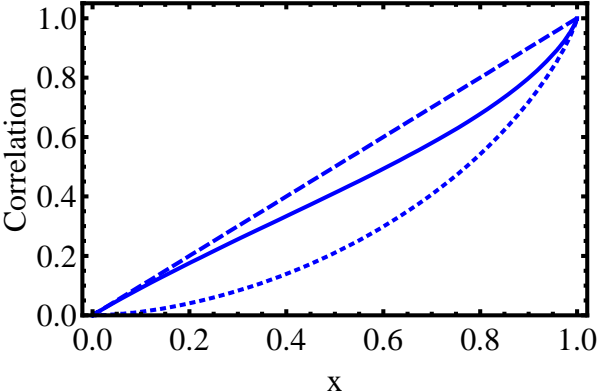

FIG. 9. Classical and quantum correlations for the initial Horodecki state. Quantum discord (solid line), classical correlation (dotted line), and concurrence (dashed line).

We display the classical correlation, quantum discord, and concurrence versus $t$ for this state in Fig. 10. Similarly to the case 5 , we can see that under decoherence the concurrence exhibits sudden death, whereas the quantum discord remains constant for asymptotic time. Moreover, the discord is always larger than the classical correlation.

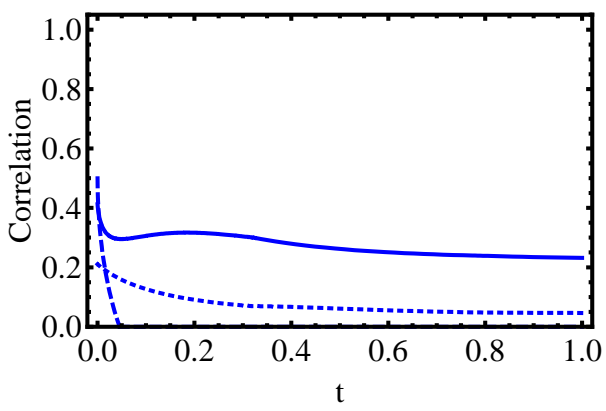

FIG. 10. Asymptotic classical and quantum correlations for the initial Horodecki state. Quantum discord (solid line), classical correlation (dotted line), and concurrence (dashed line) with $\alpha=0.5$ and $x=0.5$.

\section{CONCLUDING REMARKS}

In conclusion, we have studied the classical and quantum correlation rates in open quantum systems. We have focused on the dynamics of classical correlation, quantum discord, and entanglement of two-qubit system under dynamical semigroup generators of Kossakowskitype, which exhibits a rich manifold of asymptotic states that may be more or less correlated. Despite decoherence, the presence of an environment need not have only destructive effects in relation to classical and quantum correlations; the correlations can even be asymptotically increased with respect to the initial amount. This can be happen in the present case and the correlations generation capability of the environment is due to the nonHamiltonian contribution (10) to the generator (8). Indeed, the two-qubit Hamiltonian does not contain coupling terms and can not be a source of correlations. this can be true for (10) because the off-diagonal contributions in the Kossakowski matrix (11) couple the two qubits. This is only necessary, but not sufficient to ensure correlations generation and their asymptotic persistence. They indeed depend on a trade-off between the off-diagonal couplings and the purely decohering diagonal terms in (11).

We have evaluated the different kinds of correlations and obtained analytical expressions. The results are used to illustrate the relation between the dynamics of correlations in two-qubit sates including maximally states or partially entangled states and mixed states. We have shown that the quantum discord is more resistant to the action of the environment than the quantum entanglement and it can persist even in the asymptotic longtime regime. On the other hand, the discord is always larger than the classical correlation in this limit. Hence, the results provide the description of the behavior of the classical and quantum correlations and their robustness under the decoherence process. In fact, the quantum discord behavior between the two-qubit depends on the minimization of the classical correlations during the time evolution. This means that for initial conditions and non-Hamiltonian contribution, we expect that von Neumann measurements quantifying the classical correlations are subjected to an initial change before persisting unchanged later.

We have also noticed that, during the dissipative time evolution even without quantum entanglement, the correlations introduced by the environment are transferred to the two-qubit, producing a finite quantum discord. In fact, the quantum states with nonzero-discord are much more common than entangled states and therefore they will be easier to generate that entanglement. Furthermore, we have observed that, the classical and quantum correlations can remain unaffected for certain classes of states.

\section{Acknowledgments}

The authors would like to thank Prof. Dr. Gernot Alber, Prof. Fabio Benatti and Dr. Joseph Renes for helpful discussions. K.B. pleased to express his sincere gratitude for the hospitality at the Institut für Angewandte Physik at TU Darmstadt. The authors wish to thank the referees for their valuable comments that resulted in improvements of the paper in many aspects.

\section{APPENDIX A: CALCULATIONAL DETAILS OF THE CLASSICAL CORRELATION}

After the measurement $\left\{\Lambda_{j}\right\}$, the bipartite state $\rho(t)$ will change to the ensemble $\left\{p_{j}(t), \rho_{j}(t)\right\}$, that is $2 \times 2$ density matrices, where

$$
\rho_{j}(t):=\frac{1}{p_{j}(t)}\left(I \otimes \Lambda_{j}\right) \rho(t)\left(I \otimes \Lambda_{j}\right)
$$


with

$$
p_{j}(t)=\operatorname{tr}\left(\left(I \otimes \Lambda_{j}\right) \rho(t)\left(I \otimes \Lambda_{j}\right)\right) .
$$

In order to obtain the evolution of classical correlation, We need to evaluate $\rho_{j}(t)$ and $p_{j}(t)$. Using the von Neumann measurement $\Lambda_{j}=V \Pi_{j} V^{\dagger}$, we write

$$
p_{j}(t) \rho_{j}(t)=\left(I \otimes\left(V \Pi_{j} V^{\dagger}\right)\right) \rho(t)\left(I \otimes\left(V \Pi_{j} V^{\dagger}\right)\right) .
$$

Through some calculations, we can obtain these density matrices

$$
\begin{aligned}
& 4 p_{0}(t) \rho_{0}(t)=\left[\left(1+3 z_{3}\right) a(t)+\left(1-z_{3}\right)(1-d(t))\right]|0\rangle\langle 0| \\
& +\left[\left(1+z_{3}\right)(1-a(t))+\left(1-3 z_{3}\right) d(t)\right]|1\rangle\langle 1| \\
& +\left[2\left(z_{1}-i z_{2}\right) f(t)+\left(z_{1}+i z_{2}\right)(b(t)-c(t))\right]|1\rangle\langle 0| \\
& +\left[2\left(z_{1}+i z_{2}\right) e(t)+\left(z_{1}-i z_{2}\right)(b(t)-c(t))\right]|0\rangle\langle 1|
\end{aligned}
$$

$$
\begin{aligned}
& 4 p_{1}(t) \rho_{1}(t)=\left[\left(1-3 z_{3}\right) a(t)+\left(1+z_{3}\right)(1-d(t))\right]|0\rangle\langle 0| \\
& +\left[\left(1-z_{3}\right)(1-a(t))+\left(1+3 z_{3}\right) d(t)\right]|1\rangle\langle 1| \\
& -\left[2\left(z_{1}-i z_{2}\right) f(t)+\left(z_{1}+i z_{2}\right)(b(t)-c(t))\right]|1\rangle\langle 0| \\
& -\left[2\left(z_{1}+i z_{2}\right) e(t)+\left(z_{1}-i z_{2}\right)(b(t)-c(t))\right]|0\rangle\langle 1|,
\end{aligned}
$$

where

$z_{1}:=2\left(-t y_{2}+y_{1} y_{3}\right) ; z_{2}:=2\left(t y_{1}+y_{2} y_{3}\right) ; z_{3}:=t^{2}+y_{3}^{2}-y_{1}^{2}-y_{2}$ with

$$
z_{1}^{2}+z_{2}^{2}+z_{3}^{2}=1
$$

According to Eqs. (A4) and (A5), it is straightforward to obtain the eigenvalues characterizing the ensembles $\left\{p_{j}(t), \rho_{j}(t)\right\}$ as follows

$$
\begin{gathered}
\Gamma_{ \pm}\left(\rho_{0}(t)\right)=\frac{1}{2}[1 \pm \gamma(t)] \\
\Delta_{ \pm}\left(\rho_{1}(t)\right)=\frac{1}{2}[1 \pm \delta(t)],
\end{gathered}
$$

where we have defined $\gamma(t)$ and $\delta(t)$ as

$$
\begin{aligned}
& \gamma(t)=\left[\frac{[(a(t)-B(t)) k+(B(t)-d(t)) l]^{2}+C(t)}{[(a(t)+B(t)) k+(B(t)+d(t)) l]^{2}}\right]_{(\mathrm{A} 10)}^{\frac{1}{2}} \\
& \delta(t)=\left[\frac{[(a(t)-B(t)) l+(B(t)-d(t)) k]^{2}+C(t)}{[(a(t)+B(t)) l+(B(t)+d(t)) k]^{2}}\right]^{\frac{1}{2}}
\end{aligned}
$$

where $B(t)=(b(t)+c(t)) / 2$ and $C(t)=$ $k l[2 e(t)+b(t)-c(t)]^{2}-8 m e(t)[b(t)-c(t)], \quad$ and we have introduced the coefficients $k, l$ and $m$ as

$$
k-l=z_{3}, \quad k+l=1, \quad m=\frac{z_{2}^{2}}{4},
$$

such that

$$
k=t^{2}+y_{3}^{2} \quad l=y_{1}^{2}+y_{2}^{2} .
$$

The corresponding probabilities are given as

$$
\begin{aligned}
& p_{0}(t)=\frac{1}{2}[1+(k-l)(a(t)-d(t))] \\
& p_{1}(t)=\frac{1}{2}[1-(k-l)(a(t)-d(t))] .
\end{aligned}
$$

The entropies of the ensembles $\left\{p_{k}(t), \rho_{k}(t)\right\}$ are written as

$$
\begin{aligned}
& S\left(\rho_{0}(t)\right)=-\frac{1-\gamma(t)}{2} \log _{2} \frac{1-\gamma(t)}{2}-\frac{1+\gamma(t)}{2} \log _{2} \frac{1+\gamma(t)}{2} \\
& S\left(\rho_{1}(t)\right)=-\frac{1-\delta(t)}{2} \log _{2} \frac{1-\delta(t)}{2}-\frac{1+\delta(t)}{2} \log _{2} \frac{1+\delta(t)}{2} .
\end{aligned}
$$

Thus substituting Eqs. (A15) and (A16) into the quantum conditional entropy expression in Eq. (4), we obtain

$$
S\left(\rho(t) \mid\left\{\Lambda_{j}\right\}\right)=p_{0}(t) S\left(\rho_{0}(t)\right)+p_{1}(t) S\left(\rho_{1}(t)\right),
$$

which leads to the classical correlation

$\mathcal{C}(\rho(t))=S\left(\rho^{a}(t)\right)-\min \left[p_{0}(t) S\left(\rho_{0}(t)\right)+p_{1}(t) S\left(\rho_{1}(t)\right)\right]$

The minimal value of the $f(k, m)=S\left(\rho(t) \mid\left\{\Lambda_{j}\right\}\right)$ can be easily done analytically by appropriate choice of the parameter $k, l$, and $m$ :

$$
f(k, m)=\left\{\begin{array}{cc}
f_{\frac{1}{2}, m}(t) & \left\{m=0, \frac{1}{4}\right\} \\
f_{1, m}(t) & m=0,
\end{array}\right.
$$

then the evolution of the classical correlation can be expressed as

$$
\mathcal{C}(\rho(t))=S\left(\rho^{a}(t)\right)-\min \left[f_{\frac{1}{2}, m}(t), f_{1, m}(t)\right] .
$$

\section{APPENDIX B: CLASSICAL AND QUANTUM CORRELATIONS FOR ASYMPTOTIC STATES}

In this Appendix, we give some details of the calculations of classical and quantum correlations for asymptotic states.

When the time tends to infinity, the coefficients of the asymptotic states resulting from the inial density matrix (12) are given by

$$
\begin{aligned}
& \lim _{t \rightarrow \infty} a(t)=\frac{(1-\alpha)^{2}}{3+\alpha^{2}}(1-c), \\
& \lim _{t \rightarrow \infty} b(t)=\frac{\left(1-\alpha^{2}\right)}{3+\alpha^{2}}(1-c), \\
& \lim _{t \rightarrow \infty} d(t)=\frac{(1+\alpha)^{2}}{3+\alpha^{2}}(1-c), \\
& \lim _{t \rightarrow \infty} e(t)=\lim _{t \rightarrow \infty} f(t)=0 .
\end{aligned}
$$


which leads to the following asymptotic states

$$
\begin{aligned}
\rho(\infty) & =\frac{(1-\alpha)^{2}}{3+\alpha^{2}}(1-c)|1\rangle\left\langle 1\left|+\frac{(1+\alpha)^{2}}{3+\alpha^{2}}(1-c)\right| 2\right\rangle\langle 2| \\
& +\frac{\left(1-\alpha^{2}\right)}{3+\alpha^{2}}(1-c)|3\rangle\langle 3|+c| 4\rangle\langle 4| .
\end{aligned}
$$

There is a one-parameter set $\{\rho(\infty)\}_{0<c<1}$ of asymptotic states, where the initial states of the form (12) with the same $c$ go into the same $\rho(\infty)$. The corresponding concurrence is given by

$C(\rho(\infty))=\max \left\{0, \frac{\left|1-\alpha^{2}-4 c\right|-2\left(1-\alpha^{2}\right)(1-c)}{3+\alpha^{2}}\right\}$.

Evaluation of the classical correlation given by Eq. (A18) can obtained by taking the minimal value of the function $f$, that is, $\min \left[f_{\frac{1}{2}, 0}(\infty), f_{\frac{1}{2}, \frac{1}{4}}(\infty), f_{1,0}(\infty)\right]$.

For $k=\frac{1}{2}$, we have $\gamma(\infty)=\delta(\infty)$, and $S\left(\rho_{0}(\infty)\right)=$ $S\left(\rho_{1}(\infty)\right)$ and the minimization of $f$ is equal to the minimization of either $S\left(\rho_{0}(\infty)\right)$ or $S\left(\rho_{1}(\infty)\right)$ and is given by

$$
\begin{aligned}
f_{\frac{1}{2}, 0}(\infty)=f_{\frac{1}{2}, \frac{1}{4}}(\infty) & =-\frac{1-\gamma(\infty)}{2} \log _{2} \frac{1-\gamma(\infty)}{2} \\
& -\frac{1+\gamma(\infty)}{2} \log _{2} \frac{1+\gamma(\infty)}{2}
\end{aligned}
$$

where

$$
\gamma(\infty)=\sqrt{[a(\infty)-d(\infty)]^{2}+[b(\infty)-c]^{2}} .
$$

For $k=1$, we have

$$
f_{1,0}(\infty)=p_{0}(\infty) S\left(\rho_{0}(\infty)\right)+p_{1}(\infty) S\left(\rho_{1}(\infty)\right),
$$

where

$$
p_{0}(\infty)=\frac{1}{2}[1+(a(\infty)-d(\infty))]
$$

$$
p_{1}(\infty)=\frac{1}{2}[1-(a(\infty)-d(\infty))]
$$

$$
\begin{aligned}
S\left(\rho_{0}(\infty)\right) & =-\frac{1-\gamma(\infty)}{2} \log _{2} \frac{1-\gamma(\infty)}{2} \\
& -\frac{1+\gamma(\infty)}{2} \log _{2} \frac{1+\gamma(\infty)}{2}
\end{aligned}
$$

and

$$
\begin{aligned}
S\left(\rho_{1}(\infty)\right)= & -\frac{1-\delta(\infty)}{2} \log _{2} \frac{1-\delta(\infty)}{2} \\
& -\frac{1+\delta(\infty)}{2} \log _{2} \frac{1+\delta(\infty)}{2}
\end{aligned}
$$

with

$\gamma(\infty)=\frac{|2 a(\infty)-b(\infty)-c|}{2 a(\infty)+b(\infty)+c}, \delta(\infty)=\frac{|b(\infty)+c-2 d(\infty)|}{b(\infty)+c+2 d(\infty)}$

Based on this result, we are able to evaluate the classical correlation and quantum discord. We note that $f_{\frac{1}{2}, 0}(\infty) \leq f_{1,0}(\infty)$, that is, $\min \left[f_{\frac{1}{2}, 0}(\infty), f_{1,0}(\infty)\right]=$ $f_{\frac{1}{2}, 0}(\infty)$.

The classical correlation is given by

$$
\mathcal{C}(\rho(\infty))=S\left(\rho^{a}(\infty)\right)-f_{\frac{1}{2}, 0}(\infty)
$$

and the quantum discord is

$$
\mathcal{Q}(\rho(\infty))=S\left(\rho^{a}(\infty)\right)+f_{\frac{1}{2}, 0}(\infty)-S(\rho(\infty)) .
$$

[1] M. A. Nielsen and I. L. Chuang, Quantum Computation and Information (Cambridge University Press, Cambridge, U.K., 2000)

[2] G. Alber, T. Beth, M. Horodecki, P. Horodecki, R. Horodecki, M. Rtteler, H. Weinfurter, R. and A. Zeilinger, Quantum Information (Springer-Verlag, Berlin, 2001), Chap. 5

[3] R. Horodecki, P. Horodecki, M. Horodecki and K. Horodecki, Rev. Mod. Phys. 81, 865 (2009)

[4] D. A. Meyer, Phys. Rev. Lett. 85, 2014 (2000)

[5] M. Horodecki, P. Horodecki, R. Horodecki, J. Oppenheim, A. Sen, U. Sen and B. Synak-Radtke, Phys. Rev. A 71, 062307 (2005)

[6] A. Niset and N. J. Cerf, Phys. Rev. A 74, 052103 (2006)

[7] A. Datta, A. Shaji and C. M. Caves, Phys. Rev. Lett. 100, $050502(2008)$
[8] B. P. Lanyon, M. Barbieri, M. P. Almeida and A. G. White, Phys. Rev. Lett. 101, 200501 (2008)

[9] Jin-Shi Xu et al., Nat. Commun. 1, 7 (2010)

[10] H. Ollivier and W. H. Zurek, Phys. Rev. Lett. 88, 017901 (2001)

[11] L. Henderson and V. Vedral, J. Phys. A 34, 6899 (2001)

[12] K. Modi, T. Paterek, Wonmin, V. Vedral and M. Williamson, Phys. Rev. Lett. 104, 080501 (2010)

[13] A. Datta, e-print arXiv: 0807.4490

[14] A. Datta, A. Sheji and C. M. Caves, Phys. Rev. Lett. 100, 050502 (2008)

[15] M. Piani, P. Horodecki and R. Horodecki, Phys. Rev. Lett. 100, 090502 (2008)

[16] R. Dillenschneider, Phys. Rev. B 78, 224413 (2008)

[17] M. S. Sarardy, Phys. Rev. A 80, 022108 (2009)

[18] A. Ferraro, L. Aolita, D. Cavalcanti, F. M. Cucchietti 
and A. Acín, Phys. Rev. A 81, 052318 (2010)

[19] G. Adesso and A. Datta, Phys. Rev. Lett. 105, 030501 (2010)

[20] A. Datta and S. Gharibian, Phys. Rev. A 79, 042325 (2009)

[21] J. Cui and H. Fan, J. Phys. A 43, 045305 (2010)

[22] B. Groisman, S. Popescu and A. Winter, Phys. Rev. A 72, 032317 (2005)

[23] B. Schumacher and M. D. Westmoreland, Phys. Rev. A 74, 042305 (2006)

[24] N. Li and S. Luo, Phys. Rev. A 76, 032327 (2007)

[25] S. Luo, Phys. Rev. A 77, 042303 (2008)

[26] A. Wehrl, Rev. Mod. Phys. 50, 221 (1978)

[27] V. Vedral, Rev. Mod. Phys. 74, 197 (2002)

[28] B. Dakić, V. Vedral and C. Brukner, Phys. Rev. Lett. 105, 190502 (2010)

[29] B. Bylicka and D. Chruściński, Phys. Rev. A 81, 062102 (2010)

[30] R. Rahimi and A. Saitoh, Phys. Rev. A 82, 022314 (2010)

[31] C. Zhang, S. Yu, Q. Chen and C. H. Oh, arXiv:1005.5075

[32] A. Shabani, D. A. Lidar, Phys. Rev. Lett. 102, 100402 (2009)

[33] J. Maziero, L. C. Celeri, R. M. Serra and V. Vedral, Phys. Rev. A 80, 044102 (2009)

[34] F. F. Fanchini, T. Werlang, C. A. Brasil, L. G. E. Arruda and A. O. Caldeira, Phys. Rev. A 81, 052107 (2010)

[35] I. Chakrabarty, S. Banerjee and N. Siddharth, e-print
arXiv: 1006.1856

[36] L. Mazzola, J. Piilo and S. Maniscalco, Phys. Rev. Lett. 104, 200401 (2010)

[37] Matthias D. Lang and Carlton M. Caves, Phys. Rev. Lett. 105, 150501 (2010)

[38] J-B. Yuan, L-M. Kuang and J. Q. Liao, J. Phys. B: At. Mol. Opt. Phys. 43, 165503 (2010)

[39] M. Ali, A. R. P. Rau and G. Alber, Phys. Rev. A 81, 042105 (2010)

[40] R. Alicki and K. Lendi, Quantum Dynamical Semigroups and Applications, Lect. Notes Phys. 717 (Springer, Berlin Heidelberg 2007)

[41] H-P. Breuer and F. Petruccione, The Theory of Open Quantum Systems (Oxford: Oxford University Press 2002)

[42] F. Benatti, A. M. Liguori and G. Pluzzano, J. Phys. A: Math. Theor. 43, 045304 (2010)

[43] F. Benatti, A. M. Liguori and A. Nagy, J. Math. Phys. 49, 042103 (2008)

[44] H. Spohn, Rev. Mod. Phys. 52, 569 (1980)

[45] W. K. Wootters, Phys. Rev. Lett. 80, 2245 (1998)

[46] R. F. Werner, Phys. Rev. A 40, 4277 (1989)

[47] M. Horodecki, P. Horodecki, and R. Horodecki, in Quantum Information: An Introduction to Basic Theoretical Concepts and Experiments, edited by G. Alber et al. (Springer, Berlin, 2001), p. 151. 